\title{
Antiviral effect of pyridinium formate, a novel component of coffee extracts
}

\author{
KAZUKO TSUJIMOTO ${ }^{1,2}$, CHISEKO SAKUMA ${ }^{3}$, MISAO UOZAKI ${ }^{1}$, HISASHI YAMASAKI ${ }^{1}$, \\ HIROTOSHI UTSUNOMIYA ${ }^{2}$, KITARO OKA ${ }^{3}$ and A. HAJIME KOYAMA ${ }^{1}$ \\ ${ }^{1}$ Division of Virology, Department of Cellular and Molecular Medicine, and ${ }^{2}$ Department of \\ Strategic Surveillance for Functional Food and Comprehensive Traditional Medicine, \\ Wakayama Medical University Graduate School of Medicine, Wakayama 641-8509; \\ ${ }^{3}$ Tokyo University of Pharmacy and Life Sciences, Tokyo 192-0392, Japan
}

Received July 23, 2009; Accepted September 12, 2009

DOI: 10.3892/ijmm_00000365

\begin{abstract}
N-methyl-pyridinium formate, a novel component of coffee extracts, inhibited the multiplication of both DNA and RNA viruses. In the presence of the compound, the progeny viral yields of both herpes simplex virus type 1 (HSV-1) and poliovirus in HEp-2 cells and those of influenza virus type A in MDCK cells decreased with increasing concentrations of the compound, although the degree of viral sensitivity to this compound differed. In addition, none of these viruses were directly inactivated by the compound at the concentrations tested. Characterization of the mode of action of this compound against HSV-1 multiplication revealed that it inhibits the viral growth primarily at the initial step of virus multiplication, i.e., within $2 \mathrm{~h}$ after the onset of multiplication, although the virus multiplication was affected by the compound throughout the multiplication cycle. In addition, this compound showed a significant cytotoxic effect, although the observed antiviral effect was unlikely to be attributed to the cytotoxic effect.
\end{abstract}

\section{Introduction}

Previously, we showed that hot water extracts of coffee grinds and commercial instant coffee solutions exhibit marked antiviral activities against herpes simplex virus type 1 (HSV-1) and poliovirus (1). Characterization of the antiviral activity revealed that the activity is independent of the suppliers of

Correspondence to: Dr A. Hajime Koyama, Division of Virology, Department of Cellular and Molecular Medicine, Wakayama Medical University Graduate School of Medicine, 811-1 Kimiidera, Wakayama 641-8509, Japan

E-mail: koyama@wakayama-med.ac.jp

Key words: pyridinium formate, antiviral, herpes simplex virus, influenza virus, poliovirus, coffee the coffee grinds and of the locations where the coffee beans were produced. Instant coffee preparations also showed a similar degree of antiviral activities. The ubiquitous presence of antiviral activity among these coffee preparations suggests that the components bearing antiviral activity are stable against heating while the coffee beans are roasted.

Among the components in coffee extracts, we and others reported that caffeine exhibits an antiviral activity against HSV-1 (2-4), but there must be component(s) other than caffeine in coffee extracts which is(are) responsible for the observed antiviral activity, since a decaffeinated coffee preparation also showed a similar level of antiviral activities as regular coffee preparations (1). Considering the heatresistant nature of the antiviral activity of coffee extracts, formic acid (or its derivatives) was presumed to be a candidate antiviral substance among the components in coffee extracts, since this compound is present before the roasting process and remains even at the carbonizing step. Regarding a counter cation of formic acid, N-methyl-pyridinium is produced during roasting from trigonelline, a compound found in raw coffee beans by decarboxylation. We, therefore, examined the antiviral effects of $\mathrm{N}$-methyl-pyridinium formate on the viruses of different families (i.e., HSV-1, influenza virus and poliovirus).

\section{Materials and methods}

Cells and viruses. MDCK, HEp-2 and Vero cells were grown in Eagle's minimum essential medium (MEM) containing 5\% fetal bovine serum (FBS). Herpes simplex virus type 1 strain $\mathrm{F}$ (HSV-1), influenza virus A/Aichi/68 $\left(\mathrm{H}_{3} \mathrm{~N}_{2}\right)$ and poliovirus type 1 Sabin vaccine strain, were used as test viruses. Influenza virus was propagated in MDCK cells in MEM supplemented with $0.1 \%$ bovine serum albumin (BSA) and acetylated trypsin $(4 \mu \mathrm{g} / \mathrm{ml})$, while both HSV-1 and poliovirus were propagated in Vero cells in MEM supplemented with $0.5 \% \mathrm{FBS}$. These viruses were stored at $-80^{\circ} \mathrm{C}$ until use. The amount of each virus was measured by a plaque assay as described previously (5-7). 


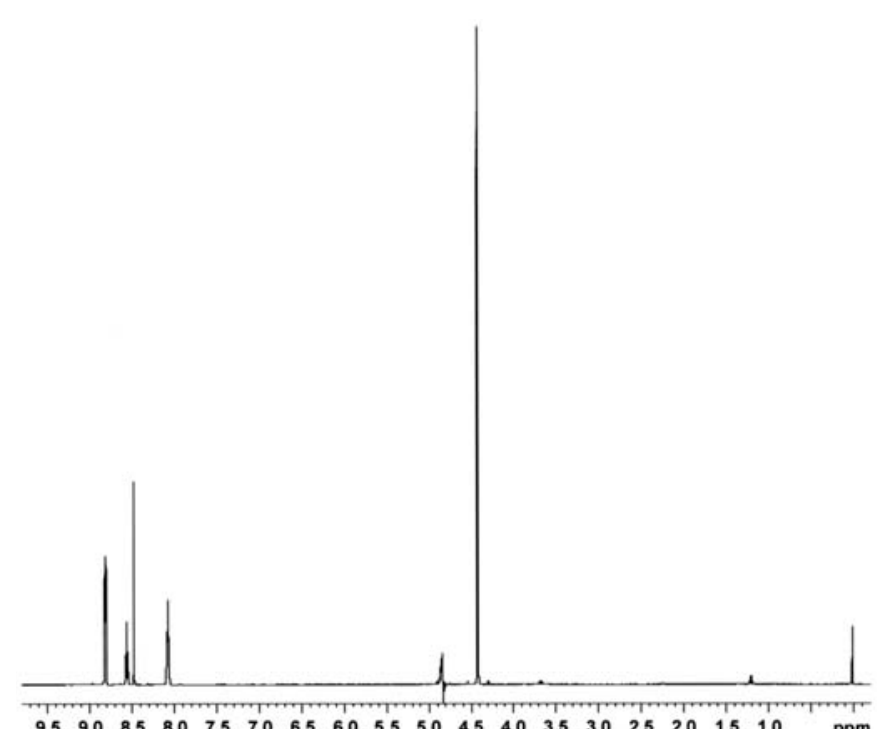

$\begin{array}{llllllllllllllllllll}9.5 & 9.0 & 8.5 & 8.0 & 7.5 & 7.0 & 6.5 & 6.0 & 5.5 & 5.0 & 4.5 & 4.0 & 3.5 & 3.0 & 2.5 & 2.0 & 1.5 & 1.0 & \mathrm{ppm}\end{array}$

Figure 1. NMR spectrum of the NMPF solutions. The NMR spectrum captured by the $600 \mathrm{MHz}$ apparatus using deuterated water containing TSP as an internal standard.

Preparation of $N$-methyl-pyridinium formate (NMPF). NMPF was synthesized as follows. Ten milliliters of a $2.0 \mathrm{M}$ aqueous solution of commercially available N-methylpyridinium chloride and the equal amount of a $2.0 \mathrm{M}$ aqueous solution of silver formate were freshly prepared. The two solutions were mixed and kept at room temperature for $1 \mathrm{~h}$. The resulting precipitates of silver chloride were removed quantitatively by filtration, and the slightly yellow colored solution containing NMPF at the concentration of $1.0 \mathrm{M}$ was obtained. As to the purity of the compound, although further purification by recrystallization was not successful, the solution showed a maximum ultraviolet absorption at $258 \mathrm{~nm}$ as expected from the structure (data not shown) and, as shown in Fig. 1, nuclear magnetic resonance (NMR) chemical shifts were observed exclusively at 4.41 (three methylprotons), 8.79 (two ortho-protons), 8.07 (two meta-protons), 8.54 (a para-proton) and $8.46 \mathrm{ppm}$ (formate proton). These spectroscopic features were consistent with the assigned structure and undeuterated hydrogens of 3-(trimethylsilyl)propionic-2,2,3,3-d4 acid sodium salt (TSP), an internal standard, and revealed that our NMPF solution did not contain possible contaminated materials except the solvent. The aqueous solution of NMPF was stored in a refrigerator until use.

Viral yields in the presence of NMPF. Monolayered cells in $35 \mathrm{~mm}$-dishes were infected with the virus at an indicated multiplicity of infection (MOI). The infected cells were further incubated at $37^{\circ} \mathrm{C}$ (for $\mathrm{HSV}-1$ and influenza virus) or $35.5^{\circ} \mathrm{C}$ (for poliovirus) for the indicated period in the serumfree MEM containing $0.1 \% \mathrm{BSA}$ and the indicated concentrations of NMPF. In the case of the influenza virus, acetylated trypsin $(4 \mu \mathrm{g} / \mathrm{ml})$ was also added to the medium for the activation of virus infectivity. At the end of infection with influenza virus, the culture medium was harvested, and the amounts of infectious virus in the medium were deter- mined as a total progeny virus. For HSV-1 and poliovirus, the amount of total progeny virus in the infected culture was determined at the indicated time after two or three cycles of freezing and thawing of the infected cells along with the culture media.

For the 'time of addition experiment', confluent monolayers of HEp-2 cells were incubated with HSV-1 for 60 min at room temperature with continuous rocking on a rocker platform (Bellco). The infection was initiated by incubating the cells at $37^{\circ} \mathrm{C}$ in MEM supplemented with $0.1 \%$ BSA. At various times after infection, NMPF $(40 \mathrm{mM})$ was added to the culture medium. At 24-h post infection (h p.i.), the infected cells together with the medium were harvested as described above, and the amounts of infectious virus in the cell lysates were assayed.

Assay for virucidal activity. All the starting materials were stored on ice prior to the virus inactivation experiments. A virus preparation $(25-\mu 1)$ was mixed with a $475-\mu 1$ aliquot of the compound solution in a $1.5-\mathrm{ml}$ plastic tube $\left[\sim 10^{8}\right.$ or $10^{9}$ plaque-forming units $\left.(\mathrm{PFU}) / \mathrm{ml}\right]$, followed by incubation at the indicated temperature. At intervals, aliquots of these virus samples were 100-fold diluted with ice-cold phosphatebuffered saline (PBS) containing 1\% calf serum (for HSV-1 or poliovirus) or $0.1 \% \mathrm{BSA}$ (for influenza virus) to stop the virucidal action of the compound. The viruses were further diluted to determine the number of infectious viruses in the treated preparation by plaque assay. All of the experiments were conducted in duplicate or triplicate $(8,9)$.

Determination of cytopathic effects (CPEs) and cell death. Monolayers of HEp-2 (for HSV-1 and poliovirus) or MDCK (for influenza virus) cells were mock-infected or infected with the test virus at the indicated MOI. The infected cells were incubated in the serum-free MEM containing $0.1 \%$ BSA and the indicated concentrations of the compound. In the case of the infection with influenza virus, the infected cells were incubated in the serum-free MEM containing $0.1 \%$ BSA and acetylated trypsin $(4 \mu \mathrm{g} / \mathrm{ml})$. The CPEs were determined by microscopic observation of the cells; approximate amounts of rounded cells on monolayers were estimated under a phase-contrast microscope.

To determine the extent of cell death in the cultures, monolayered cells were trypsinized at the indicated time p.i. to obtain a single-cell suspension. After the addition of MEM containing 5\% FBS to the suspension to neutralize the trypsin and to stabilize the cells, the numbers of the living and dead cells were determined by a dye-exclusion method with trypan blue (10).

\section{Results and discussion}

Effect of N-methyl-pyridinium formate (NMPF) on the multiplication of viruses. To examine the antiviral activities of NMPF, we tested three viruses of completely different virion structures and replication strategies; i.e., HSV-1 (Herpesviridae Family), influenza virus (Orthomyxoviridae Family) and poliovirus (Picornaviridae Family). Both HSV-1 (a double-stranded DNA virus) and influenza virus (a negative-stranded RNA virus) are large enveloped viruses 


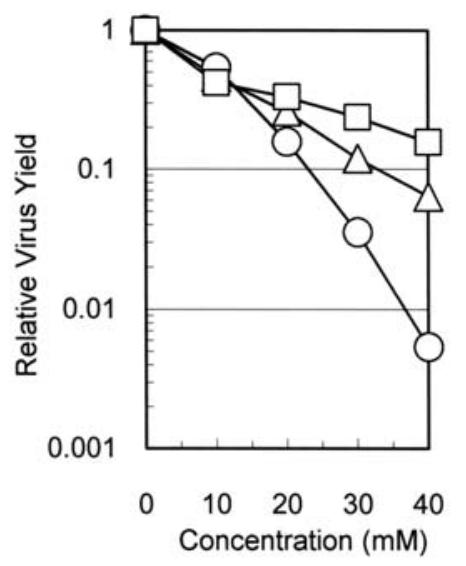

Figure 2. Effect of NMPF on the viral yields. Confluent monolayers of HEp-2 (for HSV-1 and poliovirus) or MDCK (for influenza virus) cells were infected with each of these three viruses at a MOI of 12 for HSV-1, 10 for influenza virus and 10 for poliovirus. The infected cells were incubated overnight in the medium containing varying concentrations of NMPF at $37^{\circ} \mathrm{C}$ for $\mathrm{HSV}-1$ and influenza virus or at $35.5^{\circ} \mathrm{C}$ for poliovirus. At the end of infection, the amounts of infectious progeny viruses were determined and normalized to the viral yield in the absence of the reagent. $\odot$, HSV -1 ; $\triangle$, influenza virus; $\square$, poliovirus.

and require cell nuclei for the multiplication of the virus in the infected cells $(11,12)$, while poliovirus (a positive-stranded RNA virus) is a small non-enveloped virus and replicates in the cytoplasm of the infected cells (13).

Fig. 2 shows the effects of NMPF on the relative viral yields of these three viruses, when the infected cells were incubated in the medium, which contained the indicated concentrations of the compound. The multiplication of HSV-1 in HEp-2 cells was the most sensitive to this compound; the viral yield decreased as the compound concentration was increased and, in the presence of $40 \mathrm{mM}$ NMPF, the yield decreased $>100$-fold. This decrease was 10 -fold more prominent than the decrease in the yield in the presence of $40 \mathrm{mM}$ sodium formate (data not shown), but not enough to explain the observed antiviral activity of the coffee extract (1), since the NMPF concentration in the extract was 1-2 mM at maximum, depending on the degree of roasting of the coffee beans (Oka et al, unpublished data).

In addition, the multiplication of two RNA viruses, i.e., influenza virus and poliovirus, were also affected by the compound, but were not as sensitive as HSV-1. The multiplication of the influenza virus in MDCK cells was affected concentration-dependently by the compound showing a $>10$-fold decrease in the viral yield at $40 \mathrm{mM}$ NMPF, while the multiplication of poliovirus in HEp-2 cells was less affected by the compound; the progeny viral yields of the poliovirus decreased $<10$-fold even at $40 \mathrm{mM}$ NMPF. The difference in the sensitivity to the compound between DNA and RNA viruses is not known. One possible explaination for this difference is that RNA viruses do not have a specific step in their virus multiplication which is sensitive to the compound, rather a non-specific retardation of the viral growth by NMPF occurs, while HSV-1 has a primary compound-sensitive step in the initial stage immediately after the infection, as we describe later.

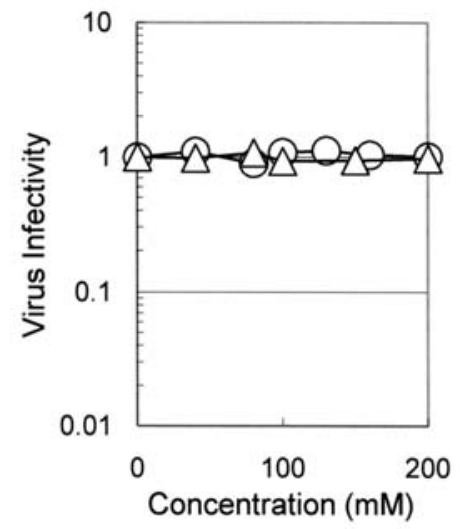

Figure 3. Direct virucidal effect of NMPF. Aliquots of the virus preparations were incubated on ice for $60 \mathrm{~min}$ in the presence of various concentrations of the reagent. The number of infectious viruses after the incubation was determined by a plaque assay method. $\odot$, HSV $-1 ; \triangle$, influenza virus.

The results, shown in Fig. 2, indicate that NMPF inhibits the multiplication of both DNA and RNA viruses, which widely differ in their virion structure (i.e., enveloped or nonenveloped) and the way genome replication and transcription occur (i.e., in the nucleus or in the cytoplasm of the infected cells). The results also revealed antiviral activity of the compound in completely different cells; MDCK cells are derived from canine kidney cells and HEp-2 cells are from human cervical carcinoma.

We previously found that coffee extracts exert, not only antiviral activity against $\mathrm{HSV}-1$, but also virucidal activity against the same virus (1). Therefore, the virucidal activity of NMPF was examined. HSV-1 or influenza virus was incubated on ice for $60 \mathrm{~min}$ with the compound at different concentrations. As shown in Fig. 3, NMPF showed no apparent virucidal activity against these two viruses. The infectivity of HSV-1 or influenza virus was not affected by the compound even at $200 \mathrm{mM}$. Similar results were obtained when the viruses were incubated at $37^{\circ} \mathrm{C}$ in the presence of the compound. This demonstrates that the observed virucidal activity of coffee extracts is due to a component (or components) other than NMPF.

Multiplication of HSV-1 in the presence of NMPF. We further characterized the mode of inhibitory action of NMPF on HSV-1 infection. Fig. 4 shows one-step growth curves of HSV-1 in HEp-2 cells in the presence or absence of NMPF. In the absence of the compound, the progeny infectious virus appeared after $6 \mathrm{~h}$ p.i. and increased rapidly for the following $8 \mathrm{~h}$, leveling off to a plateau at $\sim 14 \mathrm{~h}$ p.i. However, in the presence of NMPF at $40 \mathrm{mM}$, the virus multiplication was markedly suppressed. The formation of infectious progeny virus appeared to start at $8 \mathrm{~h}$ p.i., but then increased only slightly. The final viral yield was 100-fold less than the yield in the absence of the compound, agreeing with the result in Fig. 2. When the one-step growth curve was examined in the presence of $20 \mathrm{mM}$ NMPF, similar results were obtained except that the degree of inhibition was less prominent; the final yield was only 10 -fold less than that of the control untreated culture (data not shown). 


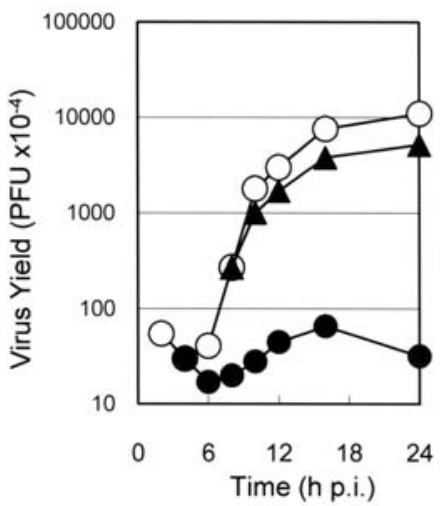

Figure 4. One-step growth curves of HSV-1 in the presence or absence of NMPF. Confluent monolayers of HEp-2 cells were infected with HSV-1 at a MOI of 17. The infected cells were incubated at $37^{\circ} \mathrm{C}$ in MEM containing $0.1 \%$ BSA ( () or in the medium additionally containing $40 \mathrm{mM} \mathrm{NMPF}(\bullet)$. Some of the dishes with the infected cells received the same concentration of the reagent at $8 \mathrm{~h}$ p.i. ( $\mathbf{\Delta})$. At the indicated time, the amounts of infectious progeny virus were determined after three cycles of freezing and thawing of the infected cells along with the culture medium.

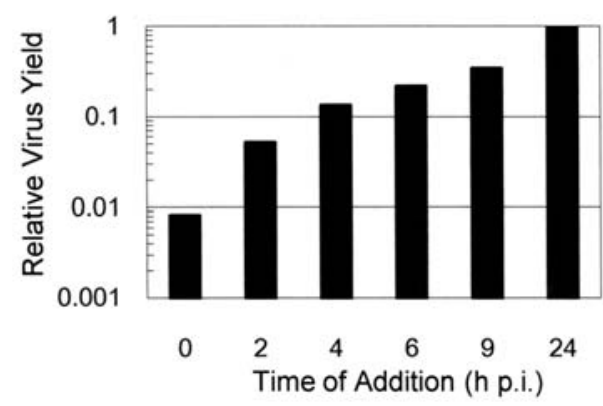

Figure 5. Effect of time of addition of NMPF on the final viral yields. HEp-2 cells, infected with HSV-1 at a MOI of 10, were incubated in MEM containing $0.1 \%$ BSA. At various times after the infection, NMPF was added to the culture medium at the final concentration of $40 \mathrm{mM}$. At $24 \mathrm{~h}$ p.i., the amount of total progeny virus in each culture was assayed. Relative viral yields in the reagent-treated cultures to that in the untreated culture were calculated. Duplicate samples for each time point.

Previously, we quantitatively characterized the kinetics of the viral DNA replication, the encapsidation of the viral DNA, the envelopment of the nucleocapsids and the formation of infectious progeny virus $(5,14)$ and revealed that viral DNA replication occurs exclusively between 3 and $6 \mathrm{~h}$ p.i. in the HSV-1-infected cells. To examine the target of NMPF, the compound was added to the infected cells at $8 \mathrm{~h}$ p.i., when the replication of viral DNA had been completed and the large amount of nucleocapsids had accumulated in the infected cells $(5,14)$. As shown in Fig. 4, the amounts of progeny virus (closed triangles) continued to increase for additional hours until the end of the virus multiplication with only a slightly reduced progeny viral yield. These results clearly demonstrate that the compound did not interfere with the virus multiplication after the viral DNA replication.

To further confirm this finding, we carried out a 'time of addition' experiment on the HSV-1-infected HEp-2 cells. After the adsorption of the virus at room temperature, the infection was initiated by incubating the cells at $37^{\circ} \mathrm{C}$, and

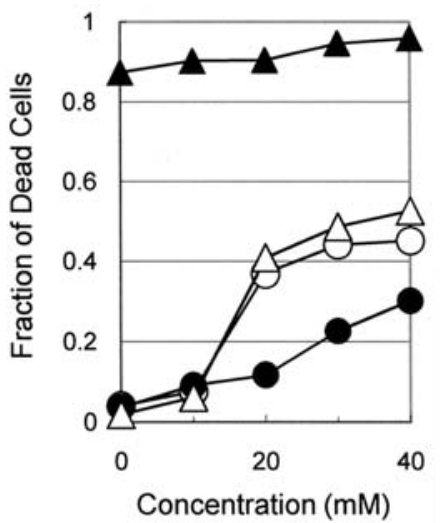

Figure 6. Effect of NMPF on the viability of infected cells. Confluent monolayers of HEp-2 $(\odot, \bullet)$ or $\operatorname{MDCK}(\triangle, \mathbf{\Delta})$ cells were mock-infected $(\odot, \triangle)$ or infected with HSV-1 at a MOI of $12(\bullet)$ or with influenza virus at a MOI of 8 ( $\mathbf{\Delta})$. The infected cells were incubated in MEM containing $0.1 \%$ BSA or in the medium additionally containing indicated concentrations of NMPF at $37^{\circ} \mathrm{C}$. At $12 \mathrm{~h}$ p.i. $(\triangle, \boldsymbol{\Delta})$ or $20 \mathrm{~h}$ p.i. $(\odot, \bullet)$, the number of dead and living cells were determined by dye-exclusion method with trypan blue.

the compound was then added to the culture medium at various times p.i. At the end of the virus multiplication ( $24 \mathrm{~h}$ p.i.), the infected culture was harvested, and the amounts of progeny viruses were determined. As shown in Fig. 5, the effect of NMPF was most prominent when added before $2 \mathrm{~h}$ p.i., although the addition at later stages of the virus multiplication was also significantly effective. Note that, prior to the addition of the compound at $0 \mathrm{~h}$ p.i., the virus adsorption had already been completed, indicating that the compound inhibits the virus multiplication at stage(s) after the virus adsorption. These results agree with the conclusions of the one-step growth experiment and indicate that there is a critical compound-sensitive step in the very initial stage (before $2 \mathrm{~h}$ p.i.) of the virus multiplication, although there is also certain compound-sensitive step(s) in the later stages of virus multiplication.

Regarding this initial stage of the HSV-1 multiplication, viral immediate early (IE) genes, such as ICP4 and ICP27, are expressed by the host RNA polymerase with the help of viral transactivator protein VP16, and these gene products play roles in this stage of the infection to positively regulate the transcription of early genes. It is of interest whether the IE genes are expressed and play roles in the presence of NMPF. Further studies may elucidate these questions.

Effect of NMPF on the infected cells. Previously, we characterized the role of virus-induced apoptosis in the multiplication of several DNA and RNA viruses and observed that the suppression of the progeny virus production by several reagents is often accompanied by a significant acceleration of virus-infected cell death $(10,15$; reviewed in refs. 16,17). Tumor necrosis factor (TNF) and other compounds including caffeine and octyl gallate showed a selective degeneration of the virus-infected cells over the uninfected cells, resulting in the abortion of the virus multiplication. In agreement with these observations, the HSV-1-infected cells showed a notable CPE (i.e., cell-rounding and detachment from the dish surface) in the presence of NMPF, while HSV-1 usually did not 
induce a significant CPE in the infected HEp-2 cells until very late in the infection.

We examined the possible selective acceleration of the infected cell death in the presence of the compound. However, as shown in Fig. 6, when the relative amount of dead cells in the HSV-1-infected HEp-2 cell culture and that of the mock-infected culture in the presence of the indicated concentrations of NMPF were examined at $20 \mathrm{~h}$ p.i., no selective degeneration by NMPF of the virus-infected cells over the uninfected cells was observed. Although the virusinfected cells in the presence of the compound showed a significantly high fraction of cell death, the amount of dead cells was much more prominent in the uninfected cells particularly at higher compound concentrations. These results indicate that NMPF has cytotoxic effects on HEp-2 cells, which can be suppressed by the infection with HSV-1, probably due to the action of the viral antiapoptosis gene.

It must be noted that the antiviral activity of NMPF is unlikely the result of the cytotoxicity of NMPF, since the addition of the compound at $2 \mathrm{~h}$ p.i. did not show a similar degree in the decrease in the viral yield at $24 \mathrm{~h}$ p.i. when compared to the addition at $0 \mathrm{~h}$ p.i. (Fig. 5); although both 0 and $2 \mathrm{~h}$ p.i. are at a very early stage in the virus multiplication. This is further supported by observations that the addition of the compound at $8 \mathrm{~h}$ p.i. (a middle stage of the infection) did not suppress the HSV-1 growth noticeably until $24 \mathrm{~h}$ p.i. (Fig. 4).

In addition, in the case of influenza virus-infected MDCK cells (Fig. 6) and poliovirus-infected Vero cells (data not shown), the acceleration of both CPE and cell death by NMPF, even if occurred, could not be detected, since these two viruses themselves induced massive CPE and cell death of the infected cells quickly after the infection. Evident from the results in Fig. 6, the selective degeneration of the infected cells is not likely to play a role in the inhibition of virus multiplication by NMPF.

In conclusion, we demonstrated that (i) the multiplication of HSV-1, influenza virus and poliovirus is sensitive to NMPF, indicating that NMPF inhibits both DNA and RNA viruses. In addition, (ii) NMPF inactivates none of these three viruses and (iii) NMPF inhibits the multiplication of HSV-1 primarily at the very initial step of the infection, although some step(s) in the late stages of virus multiplication is(are) also significantly sensitive to this reagent. (iv) Although NMPF showed a significantly cytodegenerative action, the observed antiviral effect is unlikely the result of the selective acceleration of cell death in the infected cells.

\section{Acknowledgements}

The authors thank Dr Tsutomu Arakawa (Alliance Protein Laboratory, CA) for his stimulating discussions and advice. This study was supported, in part, by a research grant from All Japan Coffee Association.

\section{References}

1. Utsunomiya H, Ichinose M, Uozaki M, Tsujimoto K, Yamasaki H and Koyama AH: Antiviral activities of coffee extracts in vitro. Food Chem Toxicol 46: 1919-1924, 2008.

2. Shiraki K and Rapp F: Effects of caffeine on herpes simplex virus. Intervirology 29: 235-240, 1988.

3. Murayama M, Tujimoto K, Uozaki M, Katsuyama Y, Yamasaki H, Utsunomiya $\mathrm{H}$ and Koyama AH: Effect of caffeine on the multiplication of DNA and RNA viruses. Mol Med Rep 1: 251-255, 2008.

4. Yamazaki Z and Tagaya I: Antiviral effect of atropine and caffeine. J Gen Virol 50: 429-431, 1980.

5. Koyama AH and Uchida T: Quantitative studies on the maturation process of herpes simplex virus type 1 in Vero cells. Virus Res 10: 281-286 1988.

6. Koyama AH, Irie H, Ueno F, Ogawa M, Nomoto A and Adachi A: Suppression of apoptotic and necrotic cell death by poliovirus. J Gen Virol 82: 2965-2972, 2001.

7. Kurokawa M, Koyama AH, Yasuoka S and Adachi A: Influenza virus overcomes apoptosis by rapid multiplication. Int J Mol Med 3: 527-530, 1999

8. Katsuyama Y, Yamasaki H, Tsujimoto K, Koyama AH, Ejima D and Arakawa T: Butyroyl-arginine as a potent virus inactivation agent. Int J Pharm 361: 92-98, 2008.

9. Yamasaki H, Tsujimoto K, Koyama AH, Ejima D and Arakawa T: Arginine facilitates inactivation of enveloped viruses. J Pharm Sci 97: 3067-3073, 2008.

10. Koyama AH, Arakawa T and Adachi A: Acceleration of virusinduced apoptosis by tumor necrosis factor. FEBS Lett 426: 179-182, 1998.

11. Roizman B and Knipe DM: Herpes simplex virus and their replication. In: Fields Virology. Fields BN, Knipe DM and Howley PM (eds). 4th edition, Lippincott-Raven, New York, pp2399-2460, 2001.

12. Lamb RA and Krug RM: Orthomyxoviridae: The viruses and their replication. In: Fields Virology. Fields BN, Knipe DM and Howley PM (eds). 4th edition, Lippincott-Raven, New York, pp1487-1530, 2001.

13. Racaniello VR: Picornaviridae: The viruses and their replication. In: Fields Virology. Fields BN, Knipe DM and Howley PM (eds). 4th edition, Lippincott-Raven, New York, pp685-722, 2001.

14. Koyama AH and Uchida T: Inhibition by Brefeldin A of the envelopment of nucleocapsids in herpes simplex virus type 1infected Vero cells. Arch Virol 135: 305-317, 1994.

15. Uozaki M, Yamasaki H, Katsuyama Y, Higuchi M, Higuchi T and Koyama AH: Antiviral effect of octyl gallate against DNA and RNA viruses. Antiviral Res 73: 85-91, 2007.

16. Koyama AH, Fukumori T, Fujita M, Irie H and Adachi A: Physiological significance of apoptosis in animal virus infection. Microbes Infect 2: 1111-1117, 2000.

17. Koyama AH, Adachi A and Irie H: Physiological significance of apoptosis during animal virus infection. Int Rev Immunol 22: 341-359, 2003. 